\title{
Treatment to prevent exacerbations in bronchiectasis: macrolides as first line?
}

\author{
Irena F. Laska (iD and James D. Chalmers \\ Affiliation: University of Dundee, Ninewells Hospital and Medical School, Dundee, UK.
}

Correspondence: James D. Chalmers, Scottish Centre for Respiratory Research, University of Dundee, Ninewells Hospital and Medical School, Dundee, DD1 9SY, UK. E-mail: jchalmersवdundee.ac.uk

@ERSpublications

Macrolides appear to be superior to inhaled corticosteroids for exacerbation prevention in bronchiectasis patients http://bit.ly/2Je3BY6

Cite this article as: Laska IF, Chalmers JD. Treatment to prevent exacerbations in bronchiectasis: macrolides as first line? Eur Respir J 2019; 54: 1901213 [https://doi.org/10.1183/13993003.01213-2019].

Bronchiectasis exacerbations are defined by an increase in daily respiratory symptoms such as cough, sputum production, malaise, fatigue and breathlessness [1-3]. Symptoms accumulate over several days and can take weeks to resolve, with many patients never fully returning to baseline after therapy [4]. Patients with frequent exacerbations experience poorer quality of life and a markedly increased mortality [5-7]. Patients tend to continue to have frequent exacerbations over time unless therapy is initiated to prevent them [5]. Bronchiectasis guidelines such as those recently issued from the European Respiratory Society (ERS) and British Thoracic Society therefore rightly prioritise exacerbation prevention as perhaps the key objective of therapy, alongside improvement of patients' symptoms and quality of life [8-10].

The ERS guidelines published in 2017 made a number of recommendations for exacerbation prevention, including recommending airway clearance and pulmonary rehabilitation, mucoactive therapy in patients who have difficulty with mucus clearance and long-term antibiotic therapy [8-11, 12]. For long-term antibiotic therapy, macrolides were recommended for patients without Pseudomonas aeruginosa infection while inhaled antibiotics were recommended for patients with $P$. aeruginosa infection [8]. Inhaled bronchodilators were recommended for breathless patients, but inhaled corticosteroids (ICS) were not recommended, with the exception of patients with co-existing asthma and chronic obstructive pulmonary disease (COPD) [8].

All of these recommendations were conditional ("we suggest" rather than "we recommend") because of a lack of randomised controlled trials for most of these therapies. Despite these recommendations and the absence of evidence, ICS remain the most widely used pharmacotherapy for patients with bronchiectasis. In the US registry, $39 \%$ of patients and in European cohorts, 55\% of patients were reported to be ICS users [13-15]. This is likely driven by the perceived overlap with other airways diseases, concerns about the adverse effects and potential development of resistance with long-term antibiotics, and about their availability, whereas ICS are largely cheap and widely available [16-20].

So how do we know which is the best maintenance therapy to introduce for the prevention of exacerbations in bronchiectasis?

In the absence of randomised clinical trials, observational data can provide important information about comparative effectiveness and safety as well as generating hypotheses that can be explored and investigated in future trials. In that regard, the contribution by HenkLe et al. [21] in this issue of the European Respiratory Journal takes us a step closer to understanding first-line therapy for bronchiectasis patients. 
They studied 618303 patients with a bronchiectasis diagnosis in the Medicare database, which provides insurance to adults over the age of 65 years. They sought to compare the relative benefit and safety of ICS and macrolides. Therefore, a subset of patients was identified and followed after the initial prescription of long-term preventative therapy; 83589 patients received ICS and 6500 received macrolides between 2006 and 2014. HeNkLE et al. [21] assessed the risk of future exacerbations and hospitalisations using these data. Physician decisions are not random, and so there are often important confounders underlying a physician decision to favour ICS or macrolide. The authors accounted for this as far as possible by using a propensity score, which adjusted for the likelihood that a physician would prescribe one or the other, to produce two cohorts matched for observable patient characteristics and risk factors.

The results show a striking advantage of macrolide treatment over ICS in terms of exacerbation reduction and prevention of severe exacerbations. Patients taking ICS were 39\% more likely to be hospitalised for respiratory infections and $56 \%$ more likely to have acute exacerbations in the adjusted models. Interestingly, there was no difference in mortality (adjusted hazard ratio 1.09), contrary to predictions given the established link between exacerbations and mortality [21].

The limitations of this type of study must be acknowledged. There is always the possibility that unmeasured confounders could account for some of the observed effect and there were differences in the groups at baseline, such as the frequency of respiratory consultations being higher in the macrolide group, that suggest possible bias by indication. The Medicare database is limited to individuals over the age of 65 years and the results of this study cannot be generalised to the whole bronchiectasis population since it is a disease that can affect patients at any age [22].

The authors interpreted their results as showing that ICS increased the risk of poor outcomes in bronchiectasis, but this study directly compared two treatments and found that macrolides were superior. This may have been due to a harmful effect of ICS, a highly beneficial effect of macrolides or a combination of both, but this study design did not allow a firm conclusion. Nevertheless, the results are plausible and consistent with what we know about both macrolide efficacy and ICS safety in bronchiectasis and other respiratory diseases [23-25]. Three randomised trials of 6 to 12 months' duration showed clear reductions in the frequency of exacerbations with macrolides, approximately halving the exacerbation rate within these populations [23-25]. A recent individual patient data meta-analysis confirmed this excellent efficacy for exacerbation reduction and demonstrated that this was consistent among nearly all patient subgroups [26]. Baseline exacerbation frequency, lung function, symptoms or quality of life did not impact upon efficacy [26].

The consistent efficacy being demonstrated for macrolides is in contrast to the data for inhaled antibiotics. The RESPIRE studies published in the ERJ in 2018 found benefit in terms of exacerbation frequency reduction of 39\% in RESPIRE 1 14-day on/off arm, but did not demonstrate clear benefit in the other three arms of the trials [27-29]. Inhaled liposomal ciprofloxacin met its primary endpoint in ORBIT 4 but not in ORBIT 3 [30]. While the pooled data showing a significant reduction in exacerbation frequency suggests this medication would be a very useful addition to the treatment of chronic P. aeruginosa infection in bronchiectasis, the inconsistency between the two trials suggests we have not yet fully identified the phenotype that best responds to inhaled antibiotics.

The data presented in this issue of the ERJ further supports the cautious use of ICS in bronchiectasis. ICS appear to increase the risk of non-tuberculous mycobacteria (NTM), promote microbial overgrowth, may adversely effect neutrophilic inflammation and, to date, have not been shown to reduce exacerbation frequency in bronchiectasis [30-33]. In COPD, where the use of ICS in combination with bronchodilators is established, the field is moving towards targeted therapy [34]. ICS are effective against eosinophilic inflammation but do not have benefits for patients with predominantly neutrophilic inflammation, and may increase bacterial load or increase the risk of pneumonia [30-37]. This is potentially of concern in patients with bronchiectasis who have predominantly neutrophilic inflammation [38]. Nevertheless, eosinophilic subtypes of bronchiectasis are starting to be identified and a potential role for anti-eosinophil therapy in selected patients has been proposed [39, 40]. It remains to be tested, but sputum or blood eosinophils may represent a potential treatable trait to guide ICS use in bronchiectasis [41]. Possible responder populations could not be explored in the study by HenkLe et al. [21] and should be the focus of future research. Bacterial load has emerged as a potential treatable trait for the guidance of inhaled antibiotic treatment [42]. Airway clearance [43,44] and macrolides in contrast appear to be almost universal in their effectiveness in the frequent exacerbator population and may be considered for the moment as the first-line pharmacological intervention.

While the evidence clearly supports the use of macrolides as the preferred treatment for exacerbation prevention in bronchiectasis, there are significant challenges. The optimal dose and dosing regimens for use in bronchiectasis have not been identified [23-26]. Gastrointestinal and other adverse effects are 


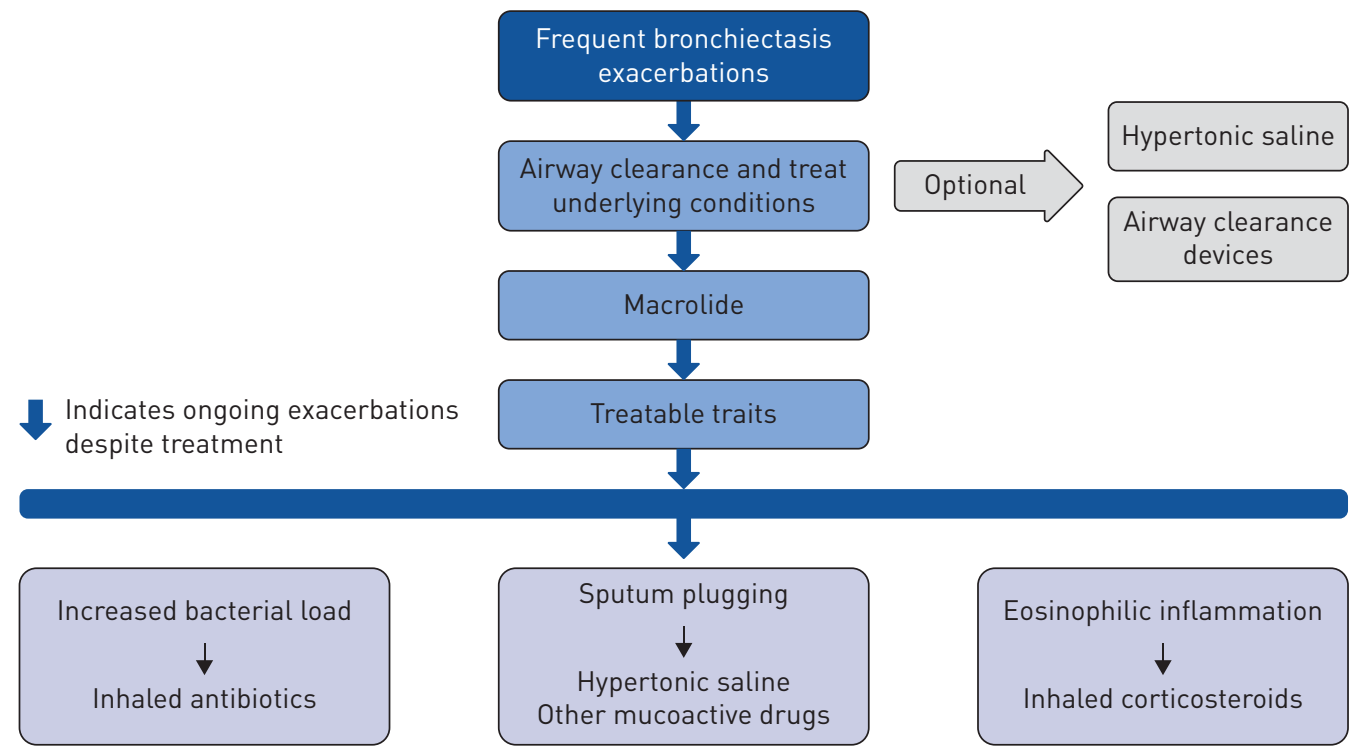

FIGURE 1 A simple schematic for exacerbation prevention in bronchiectasis.

relatively common [23-26]. Hearing loss was noted in a previous COPD trial, but this effect has not been evident in the much smaller bronchiectasis studies [45]. It nevertheless needs to be considered as a potential adverse effect in clinical practice. Antibiotic resistance emerges rapidly in the respiratory flora following macrolide treatment and changes in the microbiome are also observed, but the clinical significance of these changes in not known [23-26, 46-48]. Exclusion of non-tuberculous mycobacterial infection is recommended prior to treatment with macrolides because of the risk of inducing macrolide resistance and this is an issue of particular importance in populations with high NTM prevalence such as the USA. We have remarkably little information about what happens to patients with bronchiectasis treated with macrolides beyond the first 12 months. The data presented by HenkLE et al. [21] is relatively reassuring when comparing macrolides to other widely used drugs such as inhaled corticosteroids, but prospective studies assessing outcomes beyond 12 months are also needed.

Figure 1 shows an outline of an "integrated exacerbation prevention algorithm" based on the ERS guidelines. It is suggested that all patients should receive airway clearance and appropriate treatment of underlying causes such as immunodeficiency or allergic bronchopulmonary aspergillosis, as well as important co-morbidities. In some patients, additional airway clearance interventions may be appropriate prior to initiating prophylactic treatment with antibiotics. Macrolides may be considered the preferred option for exacerbation prevention subject to the cautions described above. In patients that continue to exacerbate despite macrolide therapy, we endorse the concept of treatable traits, which should also be considered at every step of management.

Great progress has been made in a short period of time in bronchiectasis, with recent data establishing the impact of airway clearance, macrolides and inhaled antibiotics on exacerbation prevention. Important trials of mucoactive therapies, such as the UK CLEAR study, will inform on the efficacy of hypertonic saline or carbocisteine versus usual care in the near future [49]. A search of clinical trial registries indicates one ongoing randomised trial of inhaled corticosteroids $(n=72)$ with a primary outcome of cough [50]. This study will be informative, but is unlikely to be powered to detect an effect on exacerbations or examine subgroups based on markers such as blood eosinophils. There is a clear need for a large randomised controlled trial in bronchiectasis powered for exacerbations and sufficient to identify subgroups of responders.

While awaiting such trials, the study by HenkLE et al. [21] reminds us of the value of real-life data to study drug safety and effectiveness in clinical practice.

Conflict of interest: I.F. Laska has nothing to disclose. J.D. Chalmers has received research grants from GlaxoSmithKline, Boehringer Ingelheim, AstraZeneca, Pfizer, Grifols, Bayer AG, Polyphor and Insmed; and received consultancy, congress travel or speaker fees from GlaxoSmithKline, Bayer Healthcare, Aradigm Corporation, Grifols, Pfizer, Boehringer Ingelheim, Napp and Insmed.

Support statement: This work was supported by the British Lung Foundation, GSK/BLF Chair of Respiratory Research. Funding information for this article has been deposited with the Crossref Funder Registry. 


\section{References}

1 Hill AT, Haworth CS, Aliberti S, et al. Pulmonary exacerbation in adults with bronchiectasis: a consensus definition for clinical research. Eur Respir J 2017; 49: 1700051.

2 Goeminne PC, Cox B, Finch S, et al. The impact of acute air pollution fluctuations on bronchiectasis pulmonary exacerbation: a case-crossover analysis. Eur Respir J 2018; 52: 1702557.

3 Spinou A, Siegert RJ, Guan WJ, et al. The development and validation of the Bronchiectasis Health Questionnaire. Eur Respir J 2017; 49: 1601532.

4 Brill SE, Patel AR, Singh R, et al. Lung function, symptoms and inflammation during exacerbations of non-cystic fibrosis bronchiectasis: a prospective observational cohort study. Respir Res 2015; 16: 16.

5 Chalmers JD, Aliberti S, Filonenko A, et al. Characterization of the "Frequent Exacerbator Phenotype" in bronchiectasis. Am J Respir Crit Care Med 2018; 197: 1410-1420.

6 Guan WJ, Gao YH, Xu G, et al. Inflammatory responses, spirometry, and quality of life in subjects with bronchiectasis exacerbations. Respir Care 2015; 60: 1180-1189.

7 Araujo D, Shteinberg M, Aliberti S, et al. The independent contribution of Pseudomonas aeruginosa infection to long-term clinical outcomes in bronchiectasis. Eur Respir J 2018; 51: 1701953.

8 Polverino E, Goeminne PC, McDonnell MJ, et al. European Respiratory Society guidelines for the management of adult bronchiectasis. Eur Respir J 2017; 50: 1700629.

9 Lucas JS, Barbato A, Collins SA, et al. European Respiratory Society guidelines for the diagnosis of primary ciliary dyskinesia. Eur Respir J 2017; 49: 1601090.

10 Hill AT, Sullivan AL, Chalmers JD, et al. British Thoracic Society guideline for bronchiectasis in adults. Thorax 2019; 74: Suppl. 1, 1-69.

11 Chalmers JD, Polverino E. Macrolides, mucoactive drugs and adherence for the management of bronchiectasis Eur Respir J 2018; 51: 1702033.

12 Guan WJ, Huang Y, Chen CL, et al. Macrolides, mucoactive drugs and adherence for the management of bronchiectasis. Eur Respir J 2018; 51: 1701987.

13 Aksamit TR, O'Donnell AE, Barker A, et al. Adult patients with bronchiectasis: a first look at the US Bronchiectasis Research Registry. Chest 2017; 151: 982-992.

14 Aliberti S, Polverino E, Chalmers JD, et al. The European Multicentre Bronchiectasis Audit and Research Collaboration (EMBARC) ERS Clinical Research Collaboration. Eur Respir J 2018; 52: 1802074.

15 Henkle E, Aksamit TR, Barker AF, et al. Pharmacotherapy for non-cystic fibrosis bronchiectasis: results from an NTM Info \& Research Patient Survey and the Bronchiectasis and NTM Research Registry. Chest 2017; 152: $1120-1127$.

16 Chalmers JD. Macrolide resistance in Pseudomonas aeruginosa: implications for practice. Eur Respir J 2017; 49: 1700689

17 Polverino E, Dimakou K, Hurst J, et al. The overlap between bronchiectasis and chronic airway diseases: state of the art and future directions. Eur Respir J 2018; 52: 1800328.

18 Chalmers JD, Keir HR. 10 years since TORCH: shining a new light on the risks of inhaled corticosteroids in COPD. Eur Respir J 2017; 50: 1701582.

19 Zysman M, Chabot F, Housset B, et al. Pharmacological treatment optimisation for stable COPD: an endless story? Proposals from the Société de Pneumologie de Langue Française. Eur Respir J 2017; 50: 1701250.

20 Kapur N, Petsky HL, Bell S, et al. Inhaled corticosteroids for bronchiectasis. Cochrane Database Syst Rev 2018; 5 CD000996.

21 Henkle E, Curtis JR, Chen L, et al. Comparative risks of chronic inhaled corticosteroids and macrolides for bronchiectasis. Eur Respir J 2019; 54: 1801896.

22 Chang AB, Bush A, Grimwood K. Bronchiectasis in children: diagnosis and treatment. Lancet 2018; 392: 866-879.

23 Kelly C, Chalmers JD, Crossingham I, et al. Macrolide antibiotics for bronchiectasis. Cochrane Database Syst Rev 2018; 3: CD012406.

24 Serisier DJ, Martin ML, McGuckin MA, et al. Effect of long-term, low-dose erythromycin on pulmonary exacerbations among patients with non-cystic fibrosis bronchiectasis: the BLESS randomized controlled trial. JAMA 2013; 309: 1260-1267.

25 Wong C, Jayaram L, Karalus N, et al. Azithromycin for prevention of exacerbations in non-cystic fibrosis bronchiectasis (EMBRACE): a randomised, double-blind, placebo-controlled trial. Lancet 2012; 380: 660-667.

26 Chalmers JD, Boersma W, Lonergan M, et al. Long term macrolide antibiotics for the treatment of bronchiectasis in adults- individual patient data meta-analysis. Lancet Respir Med 2019; 7: 213-226.

27 De Soyza A, Aksamit T, Bandel T-J, et al. RESPIRE 1: a phase III placebo-controlled randomised trial of ciprofloxacin dry powder for inhalation in non-cystic fibrosis bronchiectasis. Eur Respir J 2018; 51: 1702052.

28 Aksamit T, De Soyza A, Bandel T-J, et al. RESPIRE 2: a phase III placebo-controlled randomised trial of ciprofloxacin dry powder for inhalation in non-cystic fibrosis bronchiectasis. Eur Respir J 2018; 51: 1702053.

29 Chotirmall SH, Chalmers JD. RESPIRE: breathing new life into bronchiectasis. Eur Respir J 2018 ; 51 : 1702444.

30 Haworth CS, Bilton D, Chalmers JD, et al. Inhaled liposomal ciprofloxacin in patients with non-cystic fibrosis bronchiectasis and chronic lung infection with Pseudomonas aeruginosa (ORBIT-3 and ORBIT-4): two phase 3 , randomised controlled trials. Lancet Respir Med 2019; 7: 213-226.

31 Contoli M, Pauletti A, Rossi MR, et al. Long-term effects of inhaled corticosteroids on sputum bacterial and vira loads in COPD. Eur Respir J 2017; 50: 1700451.

32 Brode SK, Campitelli MA, Kwong JC, et al. The risk of mycobacterial infections associated with inhaled corticosteroid use. Eur Respir J 2017; 50: 1700037.

33 Agusti A, Fabbri LM, Singh D, et al. Inhaled corticosteroids in COPD: friend or foe? Eur Respir J 2018; 52: 1801219.

34 Wilkinson TMA. Are inhaled corticosteroids increasing the "load" for some patients with COPD? Eur Respir $J$ 2017; 50: 1701848

35 Brusselle GG, Bracke K, Lahousse L. Targeted therapy with inhaled corticosteroids in COPD according to blood eosinophil counts. Lancet Respir Med 2015; 3: 416-417.

36 Calverley PM. Through a glass darkly: inhaled corticosteroids, airway inflammation and COPD. Eur Respir J 2017; 49: 1602201 
37 Agustí A, Bafadhel M, Beasley R, et al. Precision medicine in airway diseases: moving to clinical practice. Eur Respir J 2017; 50: 1701655.

38 Flume PA, Chalmers JD, Olivier KN. Advances in bronchiectasis: endotyping, genetics, microbiome, and disease heterogeneity. Lancet 2018; 392: 880-890.

39 Chalmers JD, Chotirmall SH. Bronchiectasis: new therapies and new perspectives. Lancet Respir Med 2018; 6: 715-726.

40 Carpagnano GE, Scioscia G, Lacedonia D, et al. Severe uncontrolled asthma with bronchiectasis: a pilot study of an emerging phenotype that responds to mepolizumab. J Asthma Allergy 2019; 12: 83-90.

41 Boaventura R, Sibila O, Agusti A, et al. Treatable traits in bronchiectasis. Eur Respir J 2018; 52: 1801269.

42 Sibila O, Laserna E, Shoemark A, et al. Airway bacterial load and inhaled antibiotic response in bronchiectasis. Am J Respir Crit Care Med 2019; 200: 33-41.

43 Wong C, Sullivan C, Jayaram L. ELTGOL airway clearance in bronchiectasis: laying the bricks of evidence. Eur Respir J 2018; 51: 1702232.

44 Munoz G, de Gracia J, Buxo M, et al. Long-term benefits of airway clearance in bronchiectasis: a randomised placebo-controlled trial. Eur Respir J 2018; 51: 1701926.

45 Albert RK, Connett J, Bailey WC, et al. Azithromycin for prevention of exacerbations of COPD. N Engl J Med 2011; 365: 689-698.

46 Cox MJ, Turek EM, Hennessy C, et al. Longitudinal assessment of sputum microbiome by sequencing of the 16S rRNA gene in non-cystic fibrosis bronchiectasis patients. PLoS One 2017; 12: e0170622.

47 Rogers GB, Bruce KD, Martin ML, et al. The effect of long-term macrolide treatment on respiratory microbiota composition in non-cystic fibrosis bronchiectasis: an analysis from the randomised, double-blind, placebo-controlled BLESS trial. Lancet Respir Med 2014; 2: 988-996.

48 Mac Aogain M, Chandrasekaran R, Lim Yick Hou A, et al. Immunological corollary of the pulmonary mycobiome in bronchiectasis: the CAMEB study. Eur Respir J 2018; 52: 1800766.

49 Bradley J, Boyle R. A 2x2 factorial randomised open label trial to determine the CLinical and cost-Effectiveness of hypertonic saline (HTS 6\%) and carbocisteine for Airway cleaRance versus usual care over 52 weeks in bronchiectasis (CLEAR). https://doi.org/10.1186/ISRCTN89040295 Date last updated: March 22, 2019.

50 van der Eerden MM, de Koning Gans MJM. Formoterol-beclomethasone in Patients With Bronchiectasis: a Randomized Controlled Trial (FORZA). https://clinicaltrials.gov/ct2/show/NCT03846570 Date last updated: February 22, 2019 